\title{
ON A CONJECTURE OF GROSS
}

\author{
HUI-HSIUNG KUO ${ }^{1}$
}

ABSTRACT. Gross' conjecture about the generalized Laplacian is proved as a consequence of the main theorem.

1. Gross' conjecture. The generalized Laplacian of a Borel measurable function $f$ in an abstract Wiener space $(H, B)$ is defined by

$$
\Delta f(x)=2 \lim _{r \downarrow 0}\left\{E\left[\tau_{x}^{(r)}\right]\right\}^{-1}\left\{E\left[f\left(x+W\left(\tau_{x}^{(r)}\right)\right)\right]-f(x)\right\},
$$

where $W$ is a Wiener process in $B$ starting at the origin and $\tau_{x}^{(r)}$ is the first exit time for $x+W$ from the open ball of radius $r$ in $B$ with center $x$. Let $A$ be a bounded operator from $B$ to $B^{*}$ and $u(x)=1 / 2\langle A x, x\rangle$. Under the assumption that the $B$ norm $\|\cdot\|$ is twice continuously $B$-differentiable away from the origin and that the second Fréchet $B$-derivative is bounded on the annulus $1 \leq\|x\| \leq 2$, Gross $[2$, p. 148] showed that $\Delta u(0)=\operatorname{trace}(A \mid H)$. Then he conjectured that the conclusion remains true without any differentiability assumption on the $B$ norm or any assumption concerning the existence of smooth functions on $B$ with bounded support. The purpose of this note is to prove this conjecture. In order to use the results in [3], we assume that there exists a sequence of finite dimensional projections with range in $B^{*}$ converging strongly to the identity both in $B$ and in $H$.

2. Main theorem. We use the same notation as in [3].

Theorem 1. Let $f$ be a twice continuously $H$-differentiable function in $B$ such that $D^{2} f(x) \in \mathbb{B}_{1}(H, H)$, the Banach space of trace class operators of $H$, for all $x$ in $B$ and $D^{2} f$ is continuous from $B$ to $\mathcal{B}_{1}(H, H)$. Let $\tau$ be a stopping time (w.r.t.W) such that $\tau<\infty$ a.s. and $\int_{1}^{\infty} E\left[1_{t \leq r}\left|D f\left(x_{0}+W(t)\right)\right|^{2}\right] d t<\infty$. Then

$$
E\left[f\left(x_{0}+W(\tau)\right)\right]=f\left(x_{0}\right)+E\left[\int_{0}^{\tau} \frac{1}{2} \operatorname{trace} D^{2} f\left(x_{0}+W(t)\right) d t\right] .
$$

Received by the editors February 7, 1974 and, in revised form, May 28, 1974. AMS (MOS) subject classifications (1970)。 Primary 60H05, 60J45.

1 Research supported by NSF Grant GP-38010. 
Proof. From Ito's formula [3, Theorem 4.1]

$$
\begin{aligned}
f\left(x_{0}+W(\tau)\right)= & f\left(x_{0}\right)+\int_{0}^{\tau}\left(D f\left(x_{0}+W(t)\right), d W(t)\right) \\
& +\int_{0}^{\tau} \frac{1}{2} \text { trace } D^{2} f\left(x_{0}+W(t)\right) d t .
\end{aligned}
$$

Upon taking expectations, we obviously obtain the conclusion provided $E\left[\int_{0}^{\tau}\left(D f\left(x_{0}+W(t)\right), d W(t)\right)\right]=0$. This follows from

Lemma 1. Let $\tau$ be a stopping time and $\sigma(t)$ an $H$-valued nonanticipating process. Assume $\tau<\infty$ a.s. and $\int_{1}^{\infty} E\left[1_{t \leq \tau}|\sigma(t)|^{2}\right] d t<\infty$. Then $E\left[\int_{0}^{\tau}\left(\sigma(t), d W_{(}(t)\right)\right]=0$.

Proof. The proof of $[4, \$ 2.3(4)]$ can be easily modified to show that

$$
\int_{0}^{\tau}(\sigma(t), d W(t))=\int_{0}^{\infty}\left(1_{t \leq \tau} \sigma(t), d W(t)\right) .
$$

But

$$
\begin{aligned}
E\left[\int_{n}^{m}\left(1_{t \leq \tau} \sigma(t), d W(t)\right)\right]^{2} & =E\left[\int_{n}^{m}\left|1_{t \leq \tau} \sigma(t)\right|^{2} d t\right] \\
& =\int_{n}^{m} E\left[1_{t \leq \tau}|\sigma(t)|^{2}\right] d t \rightarrow 0 \text { as } n, m \rightarrow \infty .
\end{aligned}
$$

This gives the conclusion since $E\left[\int_{0}^{n}(\sigma(t), d W(t))\right]=0$ for any $n \geq 0$.

Corollary 1. Let $A$ be a kounded operator from $B$ to $B^{*}$ and $u(x)=$ $1 / 2\langle A x, x\rangle$. Then $\Delta u(x)=\operatorname{trace}(A \mid H)$ for all $x$ in $B$.

Proof. It is easy to see that $\left\|(A \mid H)^{*}(b)\right\|_{B^{*}} \leq\|A\|_{B, B^{*}}\|b\|$. Hence $(A \mid H)^{*}$ extends uniquely to a bounded operator from $B$ to $B^{*}$. Obviously, $D u(x)=1 / 2\left\{A x+(A \mid H)^{*} x\right\}$ and $D^{2} u(x)=1 / 2\left\{(A \mid H)+(A \mid H)^{*}\right\}$. It is well known that $(A \mid H)$ is a trace class operator of $H$, hence so is $(A \mid H)^{*}$. Let $a=$ $\|A\|_{B, H}$ and $b=\left\|(A \mid H)^{*}\right\|_{B, H}$. Then

$$
\begin{aligned}
E\left[1_{t \leq \tau_{x}^{(r)}}|D u(x+W(t))|^{2}\right] & \leq\left(a^{2}+b^{2}\right) E\left[1_{t \leq \tau_{x}^{(r)}}\|x+W(t)\|^{2}\right] \\
& \leq\left(a^{2}+b^{2}\right) r^{2} E\left[1_{t<\tau_{x}^{(r)}}\right]=\left(a^{2}+b^{2}\right) r^{2} P\left(\tau_{x}^{(r)} \geq t\right) .
\end{aligned}
$$

But $\left\{\omega ; \tau_{x}^{(r)}(\omega) \geq t\right\} \subset\{\omega ;\|W(t)\| \leq r\}$. Hence, 


$$
\begin{aligned}
E\left[1_{t \leq r_{x}^{(r)}}|D u(x+W(t))|^{2}\right] & \leq\left(a^{2}+b^{2}\right) r^{2} P(\|W(t)\| \leq r) \\
& =\left(a^{2}+b^{2}\right) r^{2} p_{t}\left(S_{r}\right)
\end{aligned}
$$

where $S_{r}=\{y ;\|y\| \leq r\}$ and $p_{t}$ is the Wiener measure.

Now, $\int_{1}^{\infty} p_{t}\left(S_{r}\right) d t \leq \int_{0}^{\infty} p_{t}\left(S_{r}\right) d t=G\left(S_{r}\right)$, which is finite by [2, Remark 3.5, p. 147]. Therefore, $u$ satisfies the assumption in Theorem 1 for all $x$ in $B$ and we have

$$
E\left[u\left(x+W\left(\tau_{x}^{(r)}\right)\right)\right]-u(x)=\frac{1}{2} E\left[\int_{0}^{\tau_{x}^{(r)}} \operatorname{trace}(A \mid H) d t\right]=\frac{1}{2} \operatorname{trace}(A \mid H) E\left[\tau_{x}^{(r)}\right] .
$$

It follows obviously that $\Delta u(x)=\operatorname{trace}(A \mid H)$ for all $x$ in $B$.

Theorem 2. Let $g$ be a twice continuously $H$-differentiable function in an open subset $U$ of $B$ such that $D^{2} g$ is continuous from $U$ to $B_{1}(H, H)$. Let $V$ be an open subset of $U$ with positive B-distance from the complement of $U$. Let $\tau$ be a stopping time sucb that

(a) almost surely, $\tau<\infty$ and $x_{0}+W(t) \in V$ for all $0 \leq t \leq \tau$, and

(b) $\int_{1}^{\infty} E\left[1_{t \leq \tau}\left|D g\left(x_{0}+W(t)\right)\right|^{2}\right] d t<\infty$.

Then

$$
E\left[g\left(x_{0}+W(\tau)\right)\right]=g\left(x_{0}\right)+E\left[\int_{0}^{\tau} \frac{1}{2} \operatorname{trace} D^{2} g\left(x_{0}+W(t)\right) d t\right]
$$

Proof. By [1, Lemma 5.4], there exists a twice $H$-differentiable function $\phi$ from $B$ to $[0,1]$ such that (i) $\phi \equiv 1$ in $V$, (ii) the support of $\phi$ has positive $B$-distance from the complement of $U$ and (iii) $D^{2} \phi$ is continuous from $B$ to $\mathcal{B}_{1}(H, H)$. This theorem follows by applying Theorem 1 to the function $f(x)=\phi(x) g(x)$.

Corollary 2. Let $g$ be a twice continuously $H$-differentiable function in an open subset $U$ of $B$ such that $D^{2} g$ is continuous from $U$ to $\Re_{1}(H, H)$. Then

$$
\Delta g(x)=\operatorname{trace} D^{2} g(x), \quad x \in U
$$

Proof. Let $x \in U$. Take $V$ to be a small ball around $x$. Then

$$
\int_{1}^{\infty} E\left[1_{t \leq \tau_{x}^{(r)}}\left|D g\left(x_{0}+W(t)\right)\right|^{2}\right] d t<\infty
$$

in Theorem 2 is satisfied by the same argument as in the proof of Corollary 1 . Hence 


$$
E\left[g\left(x+W\left(\tau_{x}^{(r)}\right)\right)\right]-g(x)=E\left[\int_{0}^{\tau_{x}^{(r)}} \frac{1}{2} \operatorname{trace} D^{2} g(x+W(t)) d t\right]
$$

The desired conclusion follows from the continuity of $D^{2} g$.

3. General theorem. Without much extra effort, Theorem 1 can be generalized to diffusion processes. Let $X(t)$ be the solution of the following stochastic integral equation:

$$
X(t)=x+\int_{0}^{t} A(X(s)) d W(s)+\int_{0}^{t} \sigma(X(s)) d s,
$$

where $A$ and $\sigma$ are given as in [3, Theorem 5.1].

Theorem 3. Let $f$ be a twice continuously $H$-differentialle function in $B$ such that $D^{2} f(x) \in B_{1}(H, H)$ for all $x$ in $B$ and $D^{2} f$ is continuous from $B$ to $\mathscr{B}_{1}(H, H)$. Let $\tau$ be a stopping time such that $\tau<\infty$ a.s. and $\int_{1}^{\infty} E\left[1_{t \leq \tau}\left|A^{*}\left(X_{t}\right) D f\left(X_{t}\right)\right|^{2}\right] d t<\infty$. Then

$$
\begin{aligned}
E[f(X(\tau))]=f(x)+E\left[\int_{0}^{\tau}\{\right. & \langle D f(X(t)), \sigma(X(t))\rangle \\
& \left.\left.+\frac{1}{2} \operatorname{trace} A^{*}(X(t)) D^{2} f(X(t)) A(X(t))\right\} d t\right] .
\end{aligned}
$$

\section{REFERENCES}

1. C. M. Elson, An extension of Weyl's lemma to infinite dimensions, Trans. Amer. Math. Soc. 194 (1974), 301-324.

2. L. Gross, Potential theory of Hilbert space, J. Functional Analysis 1 (1967), 123-181. MR 37 \#3331.

3. H.-H. Kuo, Stochastic integrals in abstract Wiener space, Pacific J. Math。 41 (1972), 469-483. MR 46 \#5561.

4. H. P. McKean, Jre, Stochastic integrals, Probability and Math. Statist., no. 5, Academic Press, New York and London, 1969. MR $40 \# 947$.

DEPARTMENT OF MATHEMATICS, UNIVERSITY OF VIRGINIA, CHARLOTTESVILLE, VIRGINIA 22903 